\title{
Evaluación del efecto de las artemisininas provenientes del extracto etanólico de Artemisia cina sobre L3 de Haemonchus contortus en una técnica de explantes abomasales
}

\section{Effect evaluation of artemisinins from ethanolic extract of Artemisia cina against L3 of Haemonchus contortus on a abomasal explants technique}

Fecha recepción: 1 de septiembre de 2015

Fecha aceptación: 17 de noviembre de 2015

\author{
Rosa Isabel Higuera-Piedrahita ${ }^{1}$ \\ María Eugenia López-Arellano ${ }^{2}$ \\ Raquel López-Arellano ${ }^{3}$ \\ César Cuenca-Verde ${ }^{4}$ \\ Jorge Alfredo Cuéllar-Ordaz ${ }^{5}$
}

\section{Resumen \\ El nuevo enfoque de control integrado de parásitos obliga a buscar alternativas que consideren el medioambiente, es decir, sostenibles y sustentables. El objetivo de este trabajo fue fraccionar el extracto etanólico de la planta Artemisia cina, obtener artemisininas y conocer el efecto de estas sobre la capaci- dad de asociación de las $L_{3}$ de Haemonchus contortus en explantes abomasales. El extracto etanólico se fraccionó por medio de la metodología establecida para Artemisia japónica, y se identificaron las artemi- sininas por medio de cromatografía en capa fina, teniendo como referencia artemisininas comerciales. Las artemisininas se utilizaron sobre $L_{3}$ desenvainada, se realizó la técnica de explantes abomasales por triplicado y se compararon así: levamisol $(7.5 \mathrm{mg} / \mathrm{ml})$, artemisinina comercial $(1 \mathrm{mg} / \mathrm{ml})$, agua y seis dife- rentes fracciones de A. cina a dosis de $1 \mathrm{mg} / \mathrm{ml}$ : Ac3k, Ac3b, Ac3a, Ac3h, Ac3i, Ac4b. No se obtuvieron diferencias significativas entre las fracciones de $A$. cina y artemisinina comercial $(p>0.05)$. Se concluye que no existió efecto de las artemisininas obtenidas del extracto etanólico de $A$. cina sobre la capacidad \\ 1 Universidad Nacional Autónoma de México (Cuautitlán Izcalli, México). \\ 2 Ph. D. Instituto Nacional de Investigaciones Forestales, Agrícolas y Pecuarias. Centro Nacional de Investigación Disci- plinaria en Parasitología Veterinaria. CENID- PAVET (Cuernavaca, México). \\ 3 Universidad Nacional Autónoma de México (Cuautitlán Izcalli, México). \\ 4 Universidad Nacional Autónoma de México (Cuautitlán Izcalli, México). \\ 5 Universidad Nacional Autónoma de México (Cuautitlán Izcalli, México). jcuellar@unam.mx.}


de asociación de las $\mathrm{L}_{3}$ de $H$. contortus al tejido abomasal. Es importante continuar con más estudios de la artemisinina para determinar sobre qué fase del parásito afecta su viabilidad.

Palabras clave: Artemisia cina; artemisinina; extracto etanólico; explantes abomasales; Haemonchus contortus.

\begin{abstract}
The new approach of integrated nematode control has driven to look for other alternatives that consider steadily and sustainable the environment. The aim of the present study was to obtain different ethanolic fracctions from Artemisia cina plant, using artemisinins, and to evaluate the nematicide association capacity effect with infective larvae $\left(L_{3}\right)$ and abomasal explant. The artemisinins from $A$. cina were obtained by thin layer chromatography and were evaluated on unsheathed $\mathrm{L}_{3}$ of Haemonchus contortus. The abomasal technique explants were performed for triplicate and compared as follows: three control with levamisole $(7.5 \mathrm{mg} / \mathrm{ml})$, commercial artemisinin $(1 \mathrm{mg} / \mathrm{ml})$, and distillate water, and six A. cina fracctions at 1 $\mathrm{mg} / \mathrm{ml}$ each one: Ac3k, Ac3b, Ac3a, Ac3h, Ac3i and Ac4b. No significant differences between fractions of $A$. cina and commercial artemisinin $(p>0.05)$ were obtained. These findings led us to conclude that there was no association capacity effect of fractions of $A$. cina on unsheathed $L_{3}$ of $H$. contortus, to the abomasal tissue. It is important to continue with more studies to determine the parasitic phase in which the artemisinin viability is susceptible to be affected.
\end{abstract}

Keywords: abomasal explants; Artemisia cina; artemisinin; ethanolic extract; Haemonchus contortus. 


\section{Introducción}

Las endoparasitosis en ovinos son de las enfermedades más impactantes en los sistemas extensivos; el tratamiento y las posibles muertes de animales susceptibles crean problemas económicos (1). $H$. contortus es el nematodo gastroentérico con mayor prevalencia en México $(2,3)$. Las deficientes prácticas actuales de manejo y control de las parasitosis han ocasionado la disminución de animales resilientes y resistentes $(4,5)$ y la mutación de cepas susceptibles a cepas resistentes de $\mathrm{H}$. contortus en los rebaños $(6,7)$.

En México se han reportado nematodos resistentes a fármacos $(8,9,10)$, y para contrarrestar esa situación se ha planteado el control integrado de parásitos, que permite utilizar muchos mecanismos que ayuden a reducir la carga parasitaria y a seleccionar los animales resilientes y resistentes $(6,11,12,13,14,15)$. Dentro de estas opciones están las plantas, cuyo efecto antiparasitario se ha corroborado, tanto por su uso tradicional como por tener algunos metabolitos con propiedades antihelmínticas $(16,17)$. Las investigaciones se han encaminado a identificar compuestos antihelmínticos para determinar su seguridad y eficacia (18). En los últimos años se ha encontrado que las plantas del género Artemisia tienen un efecto antimalárico (19), y se ha demostrado que tienen efecto antiparasitario contra el cestodo Moniezia (20); además, se han realizado estudios sobre el efecto en la $\mathrm{L}_{4}$ de $H$. contortus en gerbos (21), pero no se han realizado estudios sobre otros estadios parasíticos contra el nematodo $H$. contortus.

El objetivo de este trabajo fue fraccionar el extracto etanólico de la planta $A$. cina, obtener artemisininas y conocer su efecto sobre la capacidad de asociación de las $L_{3}$ de $H$. contortus en explantes abomasales.

\section{Materiales y métodos}

\section{Localización}

Este trabajo se realizó en la Unidad de Investigación Multidisciplinaria, laboratorio 3 y 5, de la Facultad de Estudios Superiores Cuautitlán, UNAM.
La localidad está a 2,100 msnm, tiene clima semiseco y una temperatura media anual de $16.6{ }^{\circ} \mathrm{C}$.

El fraccionamiento de la planta $A$. cina se realizó en el Centro de Investigación Científica de Yucatán (CICY), Mérida.

\section{Extracto etanólico de $\boldsymbol{A}$. cina}

Se utilizó el extracto etanólico al $96 \%$ de $A$. cina, de Laboratorio Millenium ${ }^{\circledR}$, según las reglas de la Farmacopea Homeopática Mexicana para la obtención de extractos etanólicos.

\section{Fraccionamiento de $A$. cina}

La metodología para el fraccionamiento de la planta se realizó siguiendo la descripción de Giang et al. (22) para extractos etanólicos de A. japónica.

Se realizó una partición líquido-líquido (combinación de dos moléculas inmiscibles, metanol y hexano, de forma que las moléculas polares se mezclan con el metanol y las no polares con el hexano) y se obtuvieron dos fracciones: una hexánica y una metanólica.

\section{Fracción hexánica}

De la fracción hexánica se obtuvieron cuatro fracciones (Ac2a, Ac2b, Ac3b y Ac4b) por columna de cromatografía al vacío (VLC) con tonsil. Posteriormente, se realizó una columna de gravedad, obteniéndose así catorce fracciones, identificadas como Ac3a hasta Ac3n. Las fracciones obtenidas se evaluaron por medio de cromatografía en capa fina para determinar cuál era artemisinina; y se evaluaron, además, por espectroscopia de masas (resultados no mostrados en este artículo). A las fracciones que se determinaron como artemisininas se les realizó una placa preparativa.

Placa preparativa: De la placa preparativa se obtuvieron cuatro fracciones, que fueron evaluadas para determinar artemisininas: Ac4a, Ac4b, Ac4c y Ac4d; la fracción Ac4b fue evaluada por cromatografía en capa fina y espectrometría de masas (resultados no mostrados en este estudio); se deter- 
minaron como artemisininas y se eligieron para el ensayo in vitro.

\section{Fracción metanólica}

De la fracción metanólica (de la partición líquido-líquido) se aislaron cinco fracciones. Se realizó cromatografía en capa fina para determinar artemisininas. Todas las fracciones obtenidas se liofilizaron, se mantuvieron 12 horas en desecador y se pesaron.

\section{Larvas de $\boldsymbol{H}$. contortus}

Los huevos de $H$. contortus se obtuvieron de las heces de un cordero donador, infectado experimentalmente con 5,000 larvas de tercer estadio $\left(L_{3}\right)$. La cepa es de origen ovino aislada, mantenida y caracterizada en la Facultad de Estudios Superiores Cuautitlán (23). Se colectaron larvas infectantes por medio de la técnica de Corticelli-Lai (24).

\section{Técnica de explantes abomasales}

Solo se utilizaron para el ensayo de explantes abomasales las fracciones que se determinaron como artemisininas. Se realizó según la técnica descrita por Jackson et al. (25). El abomaso se obtuvo de rastro de un animal que fue monitoreado libre de nematodos gastroentéricos. El tiempo transcurrido entre la obtención y la técnica fue de una hora. Se incidió por la curvatura menor, se retiró el contenido y se lavó con PBS a $37{ }^{\circ} \mathrm{C}$; se realizaron cortes circulares de alrededor de 3 centímetros de diámetro y se colocaron en placas de cultivo con medio Hanks a $37^{\circ} \mathrm{C}$.

A las $L_{3}$ obtenidas se les realizó el siguiente proceso:

1. Se desenvainaron con hipoclorito de sodio al $1 \%$ y se centrifugaron a 3,500 revoluciones durante cinco minutos.

2. Se realizaron tres lavados con agua destilada y se centrifugaron a 3,500 revoluciones durante cinco minutos.
3. Se aplicaron los siguientes tratamientos, como se muestra en la Tabla I:

Tabla I. Tratamientos aplicados a las $\mathrm{L}_{3}$ de $H$. contortus desenvainadas.

\begin{tabular}{ll}
\hline \multicolumn{1}{c}{ Tratamiento } & \multicolumn{1}{c}{ Dosis } \\
\hline Levamisol & $7.5 \mathrm{mg} / \mathrm{ml}$ \\
Artemisinina comercial & $1 \mathrm{mg} / \mathrm{ml}$ \\
Agua & $1 \mathrm{ml}$ \\
Ac3k & $1 \mathrm{mg} / \mathrm{ml}$ \\
Ac3b & $1 \mathrm{mg} / \mathrm{ml}$ \\
Ac3a & $1 \mathrm{mg} / \mathrm{ml}$ \\
Ac3h & $1 \mathrm{mg} / \mathrm{ml}$ \\
Ac3i & $1 \mathrm{mg} / \mathrm{ml}$ \\
Ac4b & $1 \mathrm{mg} / \mathrm{ml}$ \\
\hline
\end{tabular}

4. Se adicionaron $2,500 \pm 200 \mathrm{~L}_{3}$ de $\mathrm{H}$. contortus a cada pozo sobre el tejido abomasal.

5. Se incubaron durante tres horas y se realizaron dos lavados del tejido con PBS a $37^{\circ} \mathrm{C}$.

6. El tejido abomasal se digirió con ácido clorhídrico al $1 \%$ y pepsina al $1 \%$ durante 48 horas.

7. Se cuantificaron las $L_{3}$ asociadas al tejido.

\section{Análisis estadístico}

Los datos fueron ajustados a una curva de distribución normal por medio del programa Statgraphics $\mathrm{XV}^{\circledR}$, y posteriormente se realizó un ANOVA multifactorial por medio del mismo programa.

\section{Nota ética}

El manejo del cordero donador de huevos de $H$. contortus se realizó conforme a los lineamientos del Comité Institucional para el Cuidado y Uso de los Animales de Experimentación, de la Facultad de Estudios Superiores Cuautitlán de la Universidad Nacional Autónoma de México (CICUAEFESC-UNAM). 


\section{Resultados y discusión}

\section{Fracción hexánica}

A la fracción hexánica obtenida se le denominó Ac1a; se obtuvieron de la cromatografía al vacío con tonsil 14 subfracciones, identificadas como Ac3a hasta Ac3n (Tabla II). La fracción de la cual se obtuvo mayor peso fue la denominada Ac3k (27.9 $\mathrm{mg})$, y la de menor obtención fue la Ac3d, con un peso de $0.7 \mathrm{mg}$.

Tabla II. Subfracciones colectadas de la separación de Artemisia cina por columna de gravedad de la fracción Ac2b.

\begin{tabular}{cc}
\hline Nomenclatura & Peso $(\mathrm{mg})$ \\
\hline Ac3a & 2.8 \\
Ac3b & 1.4 \\
Ac3c & 6.9 \\
Ac3d & 0.7 \\
Ac3e & 1.3 \\
Ac3f & 1.5 \\
Ac3g & 1.2 \\
Ac3h & 0.7 \\
Ac3i & 8.8 \\
Ac3j & 13 \\
Ac3k & 27.9 \\
Ac3l & 8.8 \\
Ac3m & 3 \\
Ac3n & 1.6 \\
\hline
\end{tabular}

\section{Placa preparativa}

Se obtuvieron cuatro fracciones: Ac4a, Ac4b, Ac4c y Ac4d; de ellas, Ac4a mostró ser artemisinina (Tabla III). De la fracción Ac4b se obtuvo el mayor peso $(17.9 \mathrm{mg})$; dos fracciones tuvieron un peso de $0.1 \mathrm{mg}$.
Tabla III. Fracciones de Artemisia cina y peso, obtenidas por medio de la placa preparativa a partir de la fracción Ac3k y Ac3m.

\begin{tabular}{cc}
\hline Fracción & Peso $(\mathrm{mg})$ \\
\hline Ac4a & 8.9 \\
Ac4b & 17.9 \\
Ac4c & 0.1 \\
Ac4d & 0.1 \\
\hline
\end{tabular}

\section{Fracción metanólica}

Se obtuvieron cinco fracciones (Tabla IV), de la fracción Acb2a a la Acb2d. De la fracción más abundante (Acb2a) se obtuvieron 1,416.8 mg, y de la fracción Acb2b, 400.2 mg. Ninguna fracción obtenida de este extracto contenía artemisininas, por lo cual no se probaron in vitro.

Tabla IV. Fracciones obtenidas de la separación de Artemisia cina por medio de partición líquidolíquido en solvente metanólico.

\begin{tabular}{cc}
\hline Fracción & Peso $(\mathrm{mg})$ \\
\hline Acb2a & $1,416.8$ \\
Acb2b & 400.2 \\
Acb2c & $1,235.6$ \\
Acb2d & 61.2 \\
Total & $9,936.3$ \\
\hline
\end{tabular}

De las fracciones Ac2b y Ac2a obtenidas de $A$. cina se encontró que hay presencia de pequeñas cantidades de artemisininas, hallazgo similar al notificado por Wang et al. (26) al analizar Artemisia annua, planta de la familia Asteraceae, usada en la medicina tradicional china. La $A$. annua contiene una lactona sesquiterpénica endoperóxida, clasificada como artemisinina. La importancia de esta artemisinina en salud pública se debe a su potencial efecto contra la malaria, enfermedad causada por el protozoario Plasmodium (27). La lactona sesquiterpénica endoperoxida se encuentra en muy baja concentración en plantas que crecen bajo condiciones de trópico y subtrópico. A la fecha, no se puede determinar la forma en que se encuentra en el ambiente (28). Esos autores han sugerido que la artemisinina puede tener una presentación similar a un alcohol artemisínico, aldehído artemísico, 
aldehído dihidroartemisínico, ácido dihidroartemisínico o ácido artemisínico, y dependiendo de su presentación es el tipo de actividad que presenta en contra de patógenos. Por el proceso realizado en el presente estudio, no se determinó la forma en que se presentó, sin embargo, se logró confirmar la presencia de artemisininas.

A través de estudios se ha notificado la presencia de diversas bandas con diferente peso molecular; estos estudios incluyen procesos más detallados a nivel de resonancia magnética, $y$, por su forma, algunos autores sugieren su uso para dilucidar su posible actividad contra patógenos. Giang et al. (22) han notificado más de 500 especies del género Artemisia con gran cantidad de compuestos, entre ellos terpenoides, flavonoides, cumarinas, ácidos cafeoilquinas, poliacetilenos con variaciones oxidativas o estereoquímicas, que son probablemente compuestos obtenidos de las fracciones en estudio, pero que aún falta por caracterizar.

La metodología empleada en el presente estudio mostró la ventaja de utilizar la combinación de hexano:metanol para fraccionar y obtener mayor cantidad de compuestos presentes por polaridad. En un estudio similar, realizado por Giang et al. (22), su muestra que el mejor mecanismo para separar los compuestos de $A$. japonica era con $\mathrm{N}$-hexano, diclorometano y acetato de etilo. Este estudio buscaba separar terpenos e isómeros deri- vados de las artemisininas, al mejorar la obtención de compuestos no polares. Similar proceso fue aplicado en el presente trabajo, donde la separación de compuestos polares y no polares (hexano:metanol) permitió obtener fracciones pequeñas de artemisininas.

Como se mencionó en la metodología, los compuestos clorofílicos del extracto etanólico se retiraron por medio de tonsil, por columna de gravedad al vacío; este proceso es rápido y facilitó la separación; además, la conformación de los compuestos presentes en el extracto no fue alterada, como se corroboró en el análisis de fracciones. Con estos resultados se observó la calidad de las diferentes subfracciones analizadas para llevar a cabo los estudios de explantes abomasales (29).

\section{Explantes abomasales}

Los resultados obtenidos por los explantes abomasales de ovino se muestran en la Figura 1. Las muestras de explantes de tejido abomasal correspondientes al grupo tratado con levamisol inhibieron el $100 \%$ la invasión de $\mathrm{L}_{3}$ al tejido abomasal. Las muestras analizadas con la artemisinina comercial y la fracción Ac3h, Ac3b y Ac4b mostraron inhibición de la invasión de tejido alrededor del 30 $\%$; sin embargo, esta reducción no fue estadísticamente significativa $(p>0.05)$, pero está indicando un efecto que podría incrementar, posiblemente, por combinación de fracciones. 


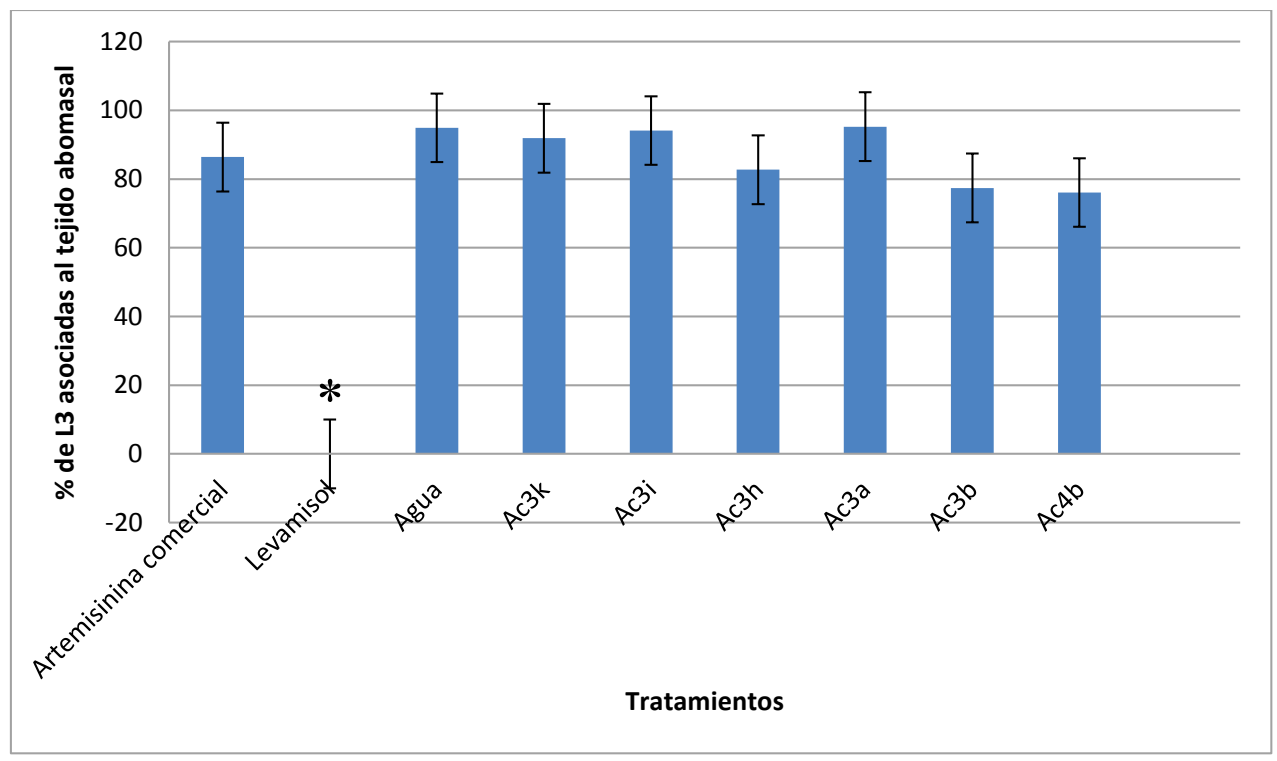

Figura 1. Proporción de $\mathrm{L}_{3}$ de $H$. contortus expuestas al levamisol y artemisininas obtenidas del extracto etanólico de $A$. cina recuperadas de la digestión del tejido abomasal.

* Porcentaje y error estándar. Diferencia significativa $(p<0.05)$.

Los resultados obtenidos de los explantes abomasales muestran, claramente, que el efecto de inhibición de $\mathrm{L}_{3}$ a tejido no incrementó como se esperaba; el efecto del levamisol es a nivel nervioso (inhibe la síntesis de acetil colinesterasa) (30). El efecto de las artemisininas podría no estar presente o en baja proporción (30 \%), como se observó en el presente estudio. Las larvas de $\mathrm{H}$. contortus colectadas postratamiento con las fracciones de $A$. cina mostraron motilidad, al igual que el control con agua; por tal motivo se considera que el bajo porcentaje del efecto nematicida de las artemisininas en contra de $L_{3}$ sin vaina de $H$. contortus requiere de otros mecanismos para inhibir la invasión de larvas al tejido, mecanismos que se deben estudiar a futuro. Las fracciones de artemisininas en estudio requieren de mayor caracterización química para poder identificar el metabolito responsable del efecto nematicida; el bajo porcentaje de eficacia podría ser enriquecido a futuro si se considera la identificación de metabolitos y estudios de sinergismo. Se debe continuar con la búsqueda del efecto nematicida sobre otro estadio parasítico.
A través de la técnica de explantes abomasales se ha evaluado la asociación de $\mathrm{L}_{3}$ de nematodos gástricos con el tejido abomasal, como un estudio de simulación in vivo (25); la asociación es un proceso importante para el establecimiento de la infección. La primera fase está representada por el establecimiento larval, donde ocurren dos pasos esenciales: el desnudamiento de larvas (eliminación de vaina) y la penetración en la mucosa abomasal a través de quimiotactismos $(\mathrm{pH}$, temperatura, enzimas, atmósfera, etc.). El papel de la asociación durante la actividad enzimática de la larva para eliminar la segunda muda inicia con la mucosa, y posteriormente se presenta la invasión a tejido (31). En el presente estudio se observó un porcentaje de asociación bajo en cuanto al mecanismo de acción de las artemisininas obtenidas del extracto etanólico de A. cina.

Se ha demostrado que las larvas de nematodos gastroentéricos de rumiantes expuestas a taninos condensados disminuyen su capacidad de asociarse al tejido en explantes abomasales (32), lo que 
no ocurrió con este estudio; por lo tanto, se descarta que su mecanismo de acción sea inhibiendo la asociación larval. Futuros estudios son necesarios para evaluar el posible mecanismo de A. cina sobre nematodos, así como para identificar el metabolito responsable de una actividad mínima, pero que podría ser relevante, considerando que es un método inocuo para los animales y sus derivados.

\section{Conclusiones}

El extracto etanólico de $A$. cina contiene pequeñas cantidades de artemisininas, aisladas por el método de cromatografía en capa fina. Se concluye que el sistema más apropiado para la colecta de artemisininas es utilizando hexano:metanol a una concentración de 80:20. Las fracciones obtenidas de A. cina no mostraron efecto potencial sobre la asociación de $\mathrm{L}_{3}$ a la mucosa abomasal como resultado de la medición por medio de explantes abomasales. Se concluye que su mecanismo de acción no es inhibir la penetración de $\mathrm{L}_{3}$ a la mucosa, posiblemente es sobre otros estadios.

\section{Agradecimientos}

Al proyecto PAPIIT/UNAM 222814, por el financiamiento de este trabajo. A la Dra. Rocío Borges Argáez, del Centro de Investigación Científica de Yucatán $(\mathrm{ClCY})$. por su apoyo técnico en el fraccionamiento de la planta Artemisia cina. A los Laboratorios Millenium ${ }^{\circledR}$, por la elaboración del extracto etanólico de Artemisia cina.

\section{Referencias}

(1) Busin V., Sargison ND. Preliminary observations on the value of using effective anthelmintic drugs to control nematode parasitism in lambs in the face of a high level of infective larval challenge. Small Ruminant Res. 2014. 119:172-175. DOI: http://dx.doi.org/10.1016/j.smallrumres.2014.02.001.

(2) Torres-Acosta JFJ, Mendoza-de-Gives P., Aguilar-Caballero AJ, Cuéllar-Ordaz JA. Anthelmintic resistance in sheep farms: update of the situation in the American continent. Vet Pa- rasitol. 2012. 189: 89-96. DOI: http://dx.doi. org/10.1016/j.vetpar.2012.03.037.

(3) Muñiz-Lagunes A., González Garduño R., López-Arellano ME, Ramírez- Valverde R., Ruíz-Flores A., García-Muñis G., Ramírez-Vargas, G., Mendoza-de Gives P., Torres-Hernández $\mathrm{G}$. Anthelmintic resistance in gastrointestinal nematodes from grazing beef cattle in Campeche State, México. Trop. Anim Health Prod. 2015. 47: 6: 1049-1054.

(4) Torres-Acosta JFJ, Jacobs DE, Aguilar-CabaIlero A., Sandoval-Castro C., Cob-Galera L., May-Martínez M. Improving resilience against natural gastrointestinal nematode infections in browsing kids during the dry season in tropical Mexico. Vet Parasitol. 2006. 135: 163-173. DOI: http://dx.doi.org/10.1016/j.vetpar.2005.08.009.

(5) Reyes J., Cuéllar-Ordaz J. Eficacia de la moxidectina, nitroxinil y rafoxanida contra nematodos gastroentéricos con resistencia múltiple a antihelmínticos en ovinos. Tesis que para obtener el título de Médico Veterinario Zootecnista. (2007). Facultad de Estudios Superiores Cuautitlán-Unam.

(6) Waller PJ. From discovery to development: current industry perspectives for the development of novel methods of helminth control in livestock. Vet Parasitol. 2006. 139: 1-14. DOI: http://dx.doi.org/10.1016/j.vetpar.2006.02.036.

(7) Klimpel S., Abdel-Ghaffar F., Al-Rasheid KA, Aksu G., Fischer K., Strassen B., Mehlhorn H. The effects of different plant extracts on nematodes. Parasitol Res. 2011. 108(4): 1047-1054. DOI: http://dx.doi.org/10.1007/s00436-0102168-4.

(8) González-Garduño R., Torres-Hernández G., Nuncio-Ochoa MGJ, Cuéllar-Ordaz JA, Zermeño-García ME. Detection of anthelmintic efficiency in nematodes of hair sheep using the faecal egg reduction test. Livestock Res. Rur. Develop. 2003. 15, 11-20. 
(9) Torres-Acosta JFJ, Dzul-Canche U., Aguilar-Caballero AJ, Rodríguez-Vivas RI. Prevalence of benzimidazole resistant nematodes in sheep flocks in Yucatan, México. Vet. Parasitol. 2003. 114: 33-42. DOI: http://dx.doi. org/10.1016/S0304-4017(03)00076-1.

(10) Nuncio-Ochoa GJ., Escobedo-Amezcua F., Morteo-Gómez R., Magaña- Damián M., González-Garduño R. Resultados preliminares de la resistencia antihelmíntica de parásitos gastrointestinales en ovinos de Tabasco. In: IV Semin. Prod. ovinos Trop. Villahermosa, Tabasco, México, 2005, pp. 100-109.

(11) Knox M., Torres-Acosta JFJ, Aguilar-Caballero A. Exploiting the effect of dietary supplementation of small ruminants on resilience and resistance against gastrointestinal nematodes. Vet. Parasitol. 2006. 139: 385-393. DOI: http://dx. doi.org/10.1016/j.vetpar.2006.04.026.

(12) Martínez-Ortiz-de-Montellano C., Vargas-Magaña JJ, Aguilar-Caballero AJ, Sandoval-Castro CA, Cob-Galera L., May-Martínez M., Miranda-Soberanis L., Hoste H., Torres-Acosta JFJ. Combining the effects of supplementary feeding and copper oxide needles improves the control of gastrointestinal nematodes in browsing goats. Vet. Parasitol. 2007. 146: 66-76. DOI: http://dx.doi.org/10.1016/j.vetpar.2007.02.012.

(13) Torres-Acosta JFJ, Hoste H. Alternative or improved methods to limit gastro-intestinal parasitism in grazing sheep and goats. Small Ruminant Res. 2008. 77: 159-173. DOI: $\quad$ http://dx.doi.org/10.1016/j.smallrumres.2008.03.009.

(14) Rocha RA, Bresciani KDS, Barros TFM, Fernandes LH, Silva MB, Amarante AFT. Sheep and cattle grazing alternately: nematode parasitism and pasture decontamination. Small Ruminant Res. 2008. 75: 135-143. DOI: http://dx.doi.org/10.1016/j.smallrumres.2007.09.001.

(15) Molento MB. Parasite control in the age of drug resistance and changing agricultural practices. Vet. Parasitol. 2009. 163: 229234. DOI: http://dx.doi.org/10.1016/j.vetpar.2009.06.007.

(16) Bar-Nun N., Mayer A.M. Cucurbitacins protect cucumber tissue against infection by Botrytis cinerea. Phytochemistry. 1990. 29: 787-791. DOI: http://dx.doi.org/10.1016/0031-9422(90)80019-D.

(17) Akhtar MS, Iqbal Z., Khan MN, Lateef M. Anthelmintic activity of medicinal plants with particular reference to their use in animals in the Indo-Pakistan subcontinent. Small Rum. Res. 2000. 38: 99-107. DOI: http://dx.doi. org/10.1016/S0921-4488(00)00163-2.

(18) Hoste H., Torres-Acosta J. Non chemical control of helminths in ruminants: adapting solutions for changing worms in a changing world. Vet. Parasitol. 2011. 180: 144-154. DOI: http:// dx.doi.org/10.1016/j.vetpar.2011.05.035.

(19) Van Agtmael MA, Eggelte TA, Boxtel CJ van. Artemisinin drugs in the treatment of malaria: from medicinal harb to registered medication. Kidlington, 1999. 20: 199-205.

(20) Bashtar A., Hassanein M., Ghaffar F., Rasheld K., Hassan S., Mehlhorn H., Al-Mahdi M., Morsy K., Ghamdi A. Studies on monieziasis of sheep I. Prevalence an antihelminthic effects of some plant extracts, a light and electron microscopic study. Parasitol. Res. 2011. 108: 177-186. DOI: http://dx.doi.org/10.1007/ s00436-010-2060-2.

(21) Squires J., Ferreira J., Lindsay D., Zajac A. Effects of artemisinin and Artemisia extracts on Haemonchus contortus in gerbils (Meriones unguiculatus). Vet. Parasitol. 2011. 175: 103-108. DOI: http://dx.doi.org/10.1016/j.vetpar.2010.09.011.

(22) Giang P., Thi N., Matsunami K., Tong P. Three new eudesmanes from Artemisia japonica. Nat. Prod. Res. 2013. 28(9): 631-635. DOI: http://dx.doi.org/10.1080/14786419.2014.89 1115. 
(23) Valdéz-Ramírez L., Cuéllar-Ordaz J. Aislamiento de una cepa de Haemonchus contortus de origen ovino. Tesis que para obtener el título de Médico Veterinario Zootecnista. Facultad de Estudios Superiores CuautitlánUnam.

(24) Van Wyk J., Mayhew E. Morphological identification of parasitic nematode infective larvae of small ruminants and cattle: A practical lab guide. J. Vet. Res. 2013. 80: 1-14. DOI: http:// dx.doi.org/10.4102/ojvr.v80i1.539.

(25) Jackson F., Greer A., Huntley J., McAnulty R., Bartley D., Stanley A., Stenhouse L., Stankiewicz M., Sykes A. Studies using Teladorsagia circumcincta in an in vitro direct challenge method using abomasal tissue explants. Vet. parasitol. 2004. 124: 73-89. DOI: http://dx. doi.org/10.1016/j.vetpar.2004.06.025.

(26) Wang Z., Yang L., Yang X., Zhan X. Synthetic communications: An international Journal for rapid communication of synthetic organic chemistry. Synthetic communications. 2014. 44(14): 1987-2003. DOI: http://dx.doi.org/10 .1080/00397911.2014.884225.

(27) Rong L., Hui-Fen D., Ming-Sen J. Artemisinin: the gifts from traditional chinesse medicine not only for malaria control but also for schistosomiasis control. Parasitol Res. 2012. 110: 2071-2074. DOI: http://dx.doi.org/10.1007/ s00436-011-2707-7.
(28) Yuan Y., Liu L., Zhang Q., Xiang L., Liu X., Chen M., Lin Z., Wang Q., Liao Z. Overexpression of artemisinic aldehyde 11 (13) reductase gene- enhanced artemisinin and its relative metabolite biosynthesis in transgenic Artemisia annua L. Biotechnol. Appl. Biochem. 2014. 62:1-17-23.

(29) Abolaji A., Eteng M., Ebong P., Dar A., Farombi E., Choudhary I. Artemisia annua as a possible contraceptive agent: a clue from mammalianrat model. Nat. Prod. Res. 2014. 28(24): 2342-2346. DOI: http://dx.doi.org/10 $.1080 / 14786419.2014 .936016$.

(30) Khöler P. The biochemical basis of anthelmintic action and resistance. Int. J. Parasitol. 2001. 31(4): 336-345. DOI: http://dx.doi. org/10.1016/S0020-7519(01)00131-X.

(31) Hoste H., Torres-Acosta JFJ. Non chemical control of helminths in ruminants: adapting solutions for changing worms in a changing world. Vet Parasitol. 2011. 180: 144-154. DOI: http:// dx.doi.org/10.1016/j.vetpar.2011.05.035.

(32) Alonso-Díaz M., Torres-Acosta C., Sandoval-Castro C., Hoste H., Aguilar- Caballero A., Capetillo-Lea C. Is goats' preference of forage trees affected by their tannin or fiber content when offered in cafeteria experiments? An. Feed Sci. Tech. 2008. 141: 36-48. DOI: http:// dx.doi.org/10.1016/j.anifeedsci.2007.04.009. 